\title{
Gain and temporal response of AIGaN solar-blind avalanche photodiodes: An ensemble Monte Carlo analysis
}

C. Sevik, and C. Bulutay

Citation: Appl. Phys. Lett. 83, 1382 (2003); doi: 10.1063/1.1602163

View online: http://dx.doi.org/10.1063/1.1602163

View Table of Contents: http://aip.scitation.org/toc/apl/83/7

Published by the American Institute of Physics

\section{Articles you may be interested in}

Solar-blind AIGaN-based Schottky photodiodes with low noise and high detectivity

Applied Physics Letters 81, 3272 (2002); 10.1063/1.1516856

Gain mechanism in GaN Schottky ultraviolet detectors

Applied Physics Letters 79, 1417 (2001); 10.1063/1.1394717

GaN avalanche photodiodes

Applied Physics Letters 76, 924 (2000); 10.1063/1.125631

High-speed, low-noise metal-semiconductor-metal ultraviolet photodetectors based on GaN

Applied Physics Letters 74, 762 (1999); 10.1063/1.123303

High quality AIGaN solar-blind Schottky photodiodes fabricated on AIN/sapphire template Applied Physics Letters 90, 121121 (2007); 10.1063/1.2715479

Compositional inhomogeneities in AIGaN thin films grown by molecular beam epitaxy: Effect on MSM UV photodetectors

Journal of Applied Physics 120, 144502 (2016); 10.1063/1.4964420

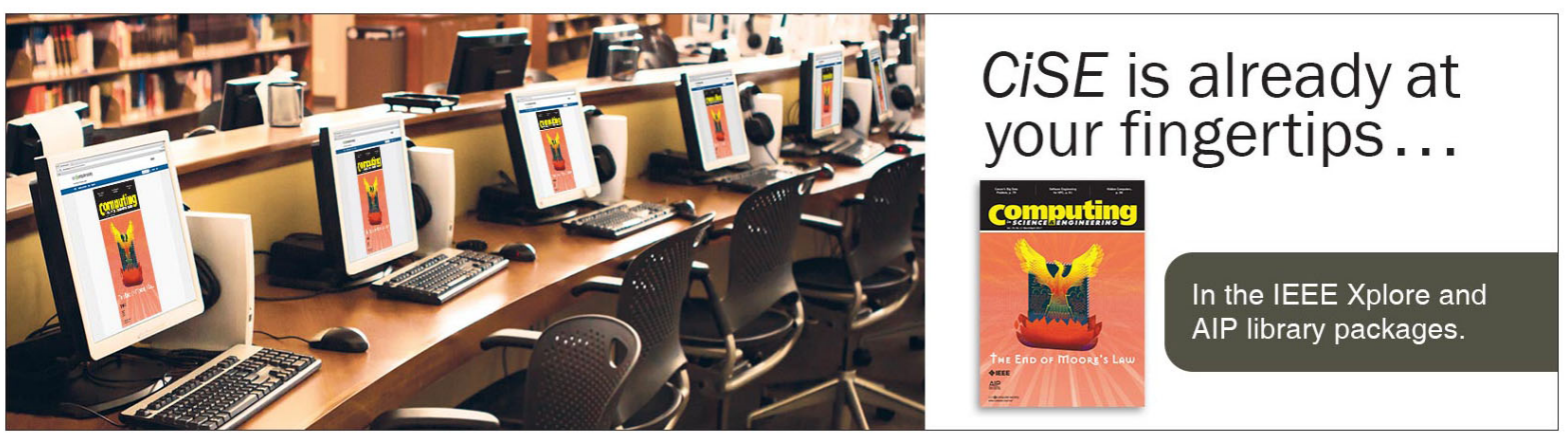




\title{
Gain and temporal response of AIGaN solar-blind avalanche photodiodes: An ensemble Monte Carlo analysis
}

\author{
C. Sevik ${ }^{\mathrm{a})}$ and C. Bulutay ${ }^{\mathrm{b})}$ \\ Department of Physics, Bilkent University, Bilkent, Ankara 06533, Turkey
}

(Received 21 April 2003; accepted 17 June 2003)

\begin{abstract}
Multiplication and temporal response characteristics of $p^{+}-n-n^{+} \mathrm{GaN}$ and $n$-type Schottky $\mathrm{Al}_{0.4} \mathrm{Ga}_{0.6} \mathrm{~N}$ avalanche photodiodes (APD) have been analyzed using the ensemble Monte Carlo method. Reasonable agreement is obtained with the published measurements for a GaN APD without any fitting parameters. In the case of AlGaN, the choice of a Schottky contact APD is seen to improve drastically the field confinement resulting in satisfactory gain characteristics. For the GaN APD, an underdamped step response is observed in the rising edge, and a Gaussian profile damping in the falling edge under an optical pulse with the switching speed degrading towards the gain region. In the $\mathrm{AlGaN}$ case, alloy scattering is seen to further slow down the temporal response while displacing the gain threshold to higher fields. (c) 2003 American Institute of Physics.
\end{abstract}

[DOI: 10.1063/1.1602163]

To obtain ultraviolet photodiodes having internal gain due to avalanche multiplication is a major objective with a potential to replace photomultiplier tube based systems for low-background applications. ${ }^{1}$ The $\mathrm{Al}_{x} \mathrm{Ga}_{1-x} \mathrm{~N}$ material with $x \geqslant 0.38$ becomes a natural candidate for the solar-blind avalanche photodiode (APD) applications which can also meet high-temperature and high-power requirements. Unfortunately, due to growth related problems, such as high defect and dislocation densities causing premature microplasma breakdown, there has been as yet no experimental demonstration of an APD with the $\mathrm{Al}_{x} \mathrm{Ga}_{1-x} \mathrm{~N}$ material. As a matter of fact, even for the relatively mature GaN-based technology, few reports of observation of avalanche gain exist. ${ }^{2-5}$

While the material quality is being gradually improved, our aim in this letter is to meet the immediate demand to explore the prospects of (Al)GaN based APDs from a computational perspective. Within the last decade, several techniques have been reported which model gain and time response of APDs. Most, however, approximate the carriers as always being at their saturated drift velocity and impact ionization rates are usually assumed to depend only on the local electric field; for references, see Ref. 6. While nonlocal effects have recently been incorporated, ${ }^{7}$ the dubious assumption on carrier drift velocity remains. Among all possible techniques, the ensemble Monte Carlo (EMC) method is potentially the most powerful as it provides a full description of the particle dynamics. However, only a small number of such simulations have been reported, predominantly on GaAs based APDs. ${ }^{8,9}$

For the high field transport phenomena, the EMC technique is currently the most reliable choice, free from major simplifications. ${ }^{10}$ All standard scattering mechanisms are included in our EMC treatment other than dislocation, neutral impurity and the piezoacoustic scatterings as they only become significant at low temperatures and fields. ${ }^{11}$ Impact ionization parameters for bulk GaN are extracted from a re-

\footnotetext{
${ }^{a)}$ Electronic mail: sevik@fen.bilkent.edu.tr

${ }^{b)}$ Electronic mail: bulutay@fen.bilkent.edu.tr
}

cent experiment of Kunihiro et al. ${ }^{12}$ As for the case of AlN, due to lack of any published results, we had to resort to a Keldysh approach, while Bloch overlaps were taken into account via the $f$-sum rule; ${ }^{13}$ for details, see Ref. 14 . Furthermore, the polar optical phonon and ionized impurity potentials are screened by using random phase approximation based dielectric function. ${ }^{15}$

The band structures for GaN and AlN are obtained using the empirical pseudopotential technique fitted to available experimental results and first principles computations. ${ }^{16,17}$ For the alloy, $\mathrm{Al}_{x} \mathrm{Ga}_{1-x} \mathrm{~N}$, we resort to linear interpolation (Vegard's Law) between the pseudopotential form factors of the constituent binaries. The necessary band edge energy, effective mass, and nonparabolicity parameters of all valleys in the lowest two conduction bands and valence bands located at high symmetry points are extracted through the computed bands of $\mathrm{GaN}$ and $\mathrm{Al}_{x} \mathrm{Ga}_{1-x} \mathrm{~N}$. To account for the remaining excited conduction and valence bands, we further append additional higher-lying parabolic free electron and hole bands. At this point it is important to stress that we use the actual density of states computed using the LehmannTaut approach, ${ }^{18}$ rather than the valley-based nonparabolic band approximation, in calculating the scattering rates. ${ }^{19}$ This assures perfect agreement with rigorous full-band EMC simulations $^{20}$ even for the hole drift velocities at a field of 1 $\mathrm{MV} / \mathrm{cm}$. To decrease the statistical noise on the current, we employ more than 60000 superparticles within the ensemble, and use the higher-order triangular-shaped-cloud representation of the superparticle charge densities. ${ }^{21}$ The Poisson solver is invoked in 0.25 fs time intervals not to cause an artificial plasma oscillation. All computations are done for a temperature of $300 \mathrm{~K}$. To avoid prolonged transients following the sudden application of a high field, the reverse dc bias is gradually applied across the APD over a linear ramp within the first 1.25 ps.

Even though, our principal aim is to characterize solarblind APDs attainable with the band gap of $\mathrm{Al}_{0.4} \mathrm{Ga}_{0.6} \mathrm{~N}$, we first test the performance of our methodology on GaN-based 

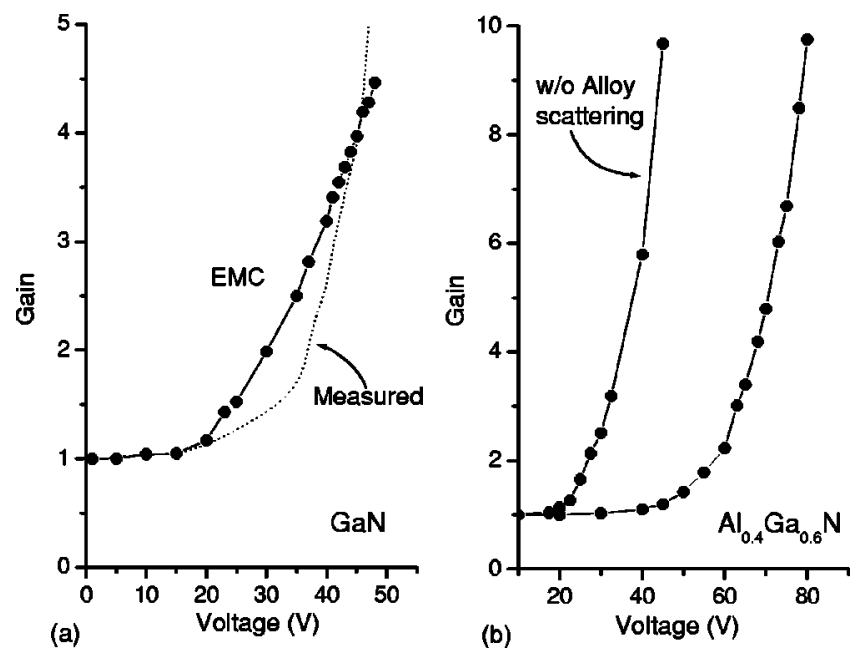

FIG. 1. (a) Current gain of the GaN APD; EMC simulation (symbols) compared with measurements (see Ref. 4) (dotted). (b) Current gain of the $\mathrm{Al}_{0.4} \mathrm{Ga}_{0.6} \mathrm{~N}$ APD simulated using EMC with and without alloy scattering. Full lines in EMC curves are used to guide the eye.

visible-blind APDs where a few experimental results have recently been reported. ${ }^{2-5}$ Among these, we choose the structure reported by Carrano et al. ${ }^{4}$ having $0.1-\mu \mathrm{m}$-thick unintentionally doped $\left(10^{16} \mathrm{~cm}^{-3}\right) n$ region sandwiched between $0.2-\mu$ m-thick heavily doped $\left(10^{18} \mathrm{~cm}^{-3}\right) p^{+}$region and a heavily doped $\left(10^{19} \mathrm{~cm}^{-3}\right) n^{+}$region. Figure 1 (a) shows that the current gain of this structure where, following Carrano et al., the current value at $1 \mathrm{~V}$ is chosen as the unity gain reference point. The overall agreement between EMC and the measurements ${ }^{4}$ is reasonable. Notably, EMC simulation yields somewhat higher values over the gain region, and the breakdown at $51 \mathrm{~V}$ cannot be observed with the simulations. Nevertheless, given the fact that there is no fitting parameter used in our simulation, we find this agreement quite satisfactory.

An important characteristic of the APDs is their time response under an optical pulse. For this purpose, an optical pump is turned on at $6.25 \mathrm{ps}$ creating electron-hole pairs at random positions consistent with the absorption profile of the electromagnetic radiation with a skin depth value of $10^{-5} \mathrm{~cm}$ for $\mathrm{GaN}$. The photon flux is assumed to be such that an electron-hole pair is created in 0.5 fs time intervals. The optical pump is kept on for 25 ps to assure that steady state is attained and afterwards it is turned off at 31.25 ps to observe the fall of the current. As we are assuming a $p$-side illumination, it is mainly the electrons which travel through the multiplication region, even though in the simulation the impact ionization of both electrons and holes are included. The falling edge of the optical pulse response can be fitted with a Gaussian profile $\exp \left(-t^{2} / \tau_{f}^{2}\right)$, see parameters in Table I. As seen in Fig. 2 and Table I, the width of the Gaussian profile increases with the applied bias. Hence, the temporal

TABLE I. Fitted temporal response functions $\exp \left(-t^{2} / \tau_{f}^{2}\right)$ and $1-\exp \left(-t^{2} / \tau_{r}^{2}\right) \cos \left(\omega_{r} t\right)$ for the GaN APD.

\begin{tabular}{cccc}
\hline \hline Bias $(\mathrm{V})$ & $\tau_{f}(\mathrm{ps})$ & $\tau_{r}(\mathrm{ps})$ & $\omega_{r}(\mathrm{r} / \mathrm{ps})$ \\
\hline 25 & 1.72 & 3.06 & 0.35 \\
30 & 1.78 & 2.57 & 0.38 \\
40 & 2.04 & 1.75 & 0.506 \\
\hline \hline
\end{tabular}

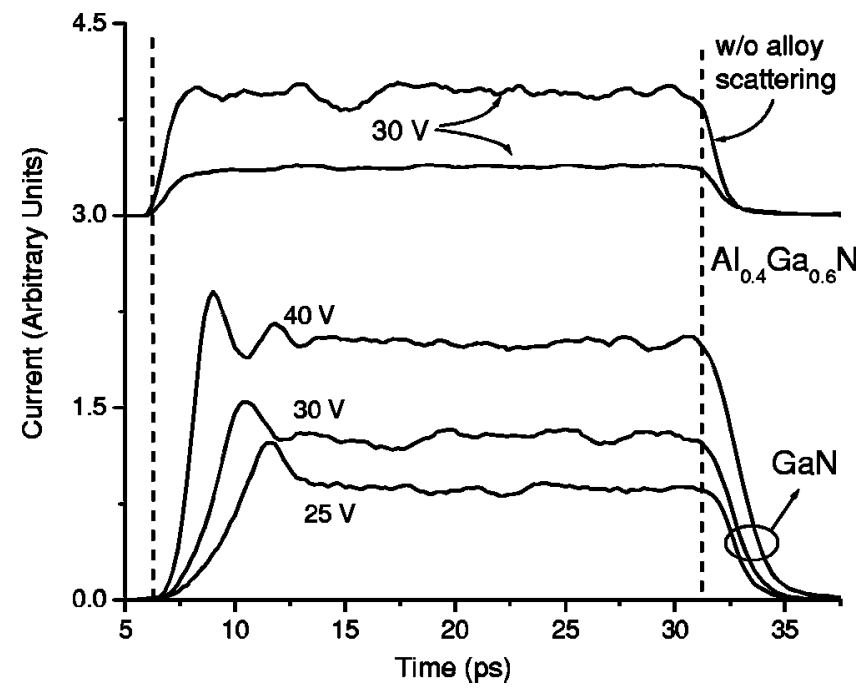

FIG. 2. Temporal response of the GaN and $\mathrm{Al}_{0.4} \mathrm{Ga}_{0.6} \mathrm{~N}$ (vertically shifted for clarity) APD to a 25 ps optical pulse, applied between the dashed lines.

response of the device degrades in the high gain region where substantial amount of secondary carriers exist, as expected. The rising edge of the pulse shows an underdamped behavior, becoming even more pronounced towards the gain region; this can approximately be fitted by a function $1-\exp \left(-t^{2} / \tau_{r}^{2}\right) \cos \left(\omega_{r} t\right)$, with the parameters being listed in Table I.

Figure 3(a) demonstrates the electric field profile of the $\mathrm{GaN}$ APD. Observe that as the applied bias increases the moderately doped $p^{+}$region becomes vulnerable to the penetration of the electric field, hence, preventing further building up in the multiplication region and increasing the impact ionization events. In this regard, it needs to be mentioned that achieving very high $p$ doping persists as a major technological challenge. Therefore, in our considerations to follow for the AlGaN APDs, we replace the problematic $p^{+}$ region with a Schottky contact. So, we analyze an $\mathrm{Al}_{0.4} \mathrm{Ga}_{0.6} \mathrm{~N}$ APD of $0.1-\mu \mathrm{m}$-thick unintentionally doped $\left(10^{16} \mathrm{~cm}^{-3}\right) n$ region sandwiched between a Schottky contact and a heavily doped $\left(10^{19} \mathrm{~cm}^{-3}\right) n^{+}$region. Previously, in unipolar $\mathrm{AlGaN}$ structures we observed the alloy scattering to be substantial, ${ }^{22}$ whereas the actual significance of this
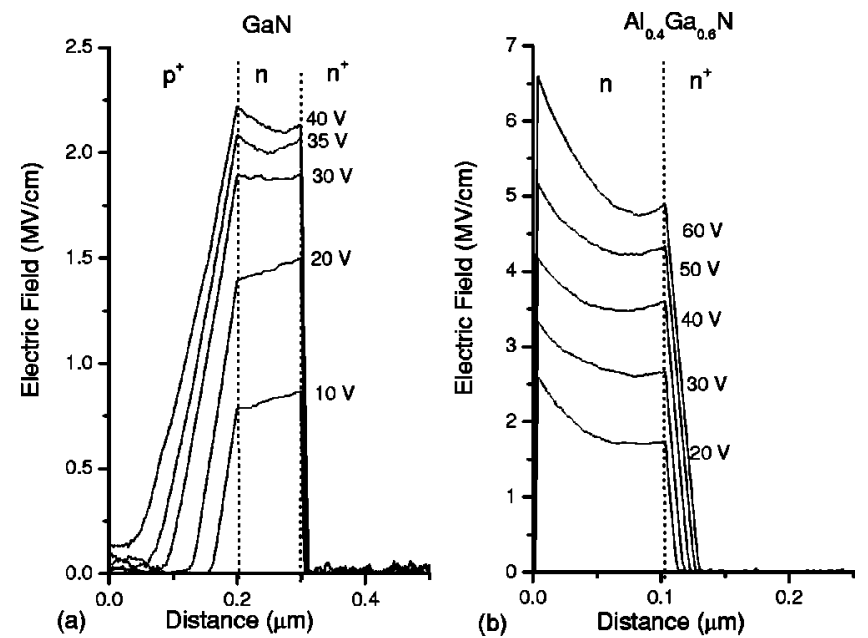

FIG. 3. (a) Electric field distribution over (a) $\mathrm{GaN}$ (b) $\mathrm{Al}_{0.4} \mathrm{Ga}_{0.6} \mathrm{~N}$ APDs at several bias levels. 
TABLE II. Fitted temporal response functions $\exp \left(-t / \tau_{f}\right)$ and $1-\exp \left(-t^{2} / \tau_{r}^{2}\right)$ for $\mathrm{Al}_{0.4} \mathrm{Ga}_{0.6} \mathrm{~N}$ APD under a reverse bias of $30 \mathrm{~V}$.

\begin{tabular}{ccc}
\hline \hline Alloy scattering & $\tau_{f}(\mathrm{ps})$ & $\tau_{r}(\mathrm{ps})$ \\
\hline No & 0.75 & 0.67 \\
Yes & 1.06 & 2.14 \\
\hline
\end{tabular}

mechanism has always been controversial. ${ }^{13}$ For this reason, we provide in Fig. 1(b) the gain characteristics of this $\mathrm{Al}_{0.4} \mathrm{Ga}_{0.6} \mathrm{~N}$ APD both with and without alloy scattering. The presence of alloy scattering almost doubles the breakdown voltage with respect to the case when there is no alloy scattering. The time response of the $\mathrm{Al}_{0.4} \mathrm{Ga}_{0.6} \mathrm{~N}$ APD is shown in Fig. 2 in the low gain region $(30 \mathrm{~V})$ under the same optical illuminations discussed in the GaN case, above. The falling edge of the response can be fitted by an exponential $\exp \left(-t / \tau_{f}\right)$ whereas the rising edge by a Gaussian function, $1-\exp \left(-t^{2} / \tau_{r}^{2}\right)$; see Table II for the parameters.

Figure 3(b) demonstrates the electric field profile of this $\mathrm{Al}_{0.4} \mathrm{Ga}_{0.6} \mathrm{~N}$ structure. It is observed that for all values of the applied bias, the electric field is confined in the intrinsic (multiplication) region which is very desirable for the APD operation. Finally, we would like to check the standard assumption made in other theoretical APD treatments assuming the carriers to travel at their saturated drift velocities. It is seen in Fig. 4 that this assumption may be acceptable for the
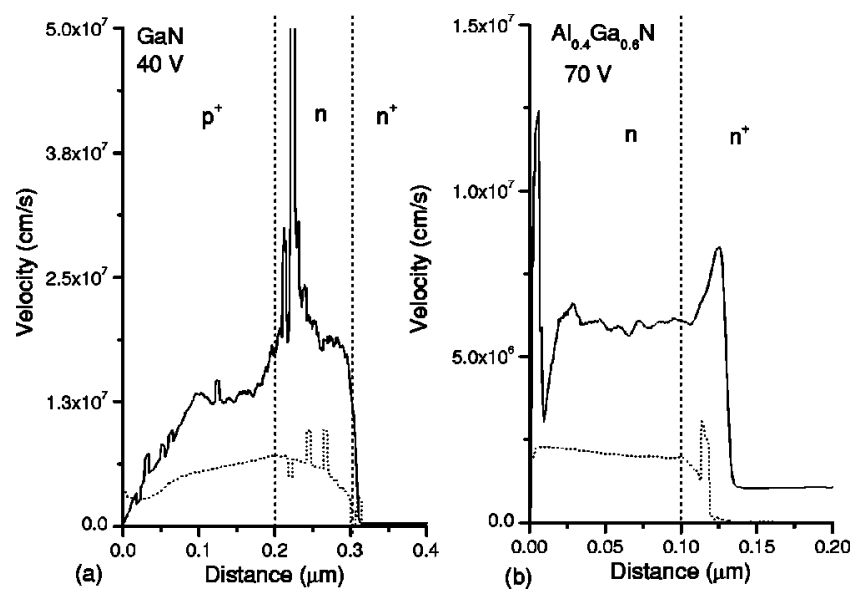

FIG. 4. Average velocity distribution over (a) $\mathrm{GaN}$ (b) $\mathrm{Al}_{0.4} \mathrm{Ga}_{0.6} \mathrm{~N}$ APDs for electron (solid) and holes (dotted).
Schottky $\mathrm{Al}_{0.4} \mathrm{Ga}_{0.6} \mathrm{~N}$ APD, whereas it is not appropriate in the $\mathrm{GaN}$ case.

In summary, gain, electric field, and velocity profiles as well as the time response of the $\mathrm{GaN}$ and $\mathrm{Al}_{0.4} \mathrm{Ga}_{0.6} \mathrm{~N}$ APDs are computed by the EMC method. Results for the $\mathrm{Al}_{0.4} \mathrm{Ga}_{0.6} \mathrm{~N}$ APDs are provided both with and without the alloy scattering. In this respect, experimental reports will be invaluable to resolve the actual significance of alloy scattering and also to assess the effects of dislocations or other defects which were unaccounted in our analysis.

The authors would like to thank The Scientific and Technical Research Council of Turkey (TÜBITAK) and Bilkent University for their financial support. The authors gratefully acknowledge the continuous encouragement of Professor B. Tanatar and stimulating discussions with N. Bıyıklı.

${ }^{1}$ M. Razeghi, Proc. IEEE Proc. IEEE 90, 1006 (2002).

${ }^{2}$ A. Osinsky, M. S. Shur, R. Gaska, and Q. Chen, Electron. Lett. 34, 691 (1998).

${ }^{3}$ K. A. McIntosh, R. J. Molnar, L. J. Mahoney, A. Lightfoot, M. W. Geis, K. M. Molvar, I. Melngailis, R. L. Aggarwal, W. D. Goodhue, S. S. Choi, D. L. Spears, and S. Verghese, Appl. Phys. Lett. 75, 3485 (1999).

${ }^{4}$ J. C. Carrano, D. J. H. Lambert, C. J. Eiting, C. J. Collins, T. Li, S. Wang, B. Yang, A. L. Beck, R. D. Dupuis, and J. C. Campbell, Appl. Phys. Lett. 88, 924 (2000).

${ }^{5}$ B. Yang, T. Li, K. Heng, C. Collins, S. Wang, J. C. Carrano, R. D. Dupuis, R. C. Campbell, M. J. Schurman, and I. T. Ferguson, IEEE J. Quantum Electron. 36, 1389 (2000)

${ }^{6}$ P. J. Hambleton, S. A. Plimmer, J. P. R. David, G. J. Rees, and G. M. Dunn, J. Appl. Phys. 91, 2107 (2002).

${ }^{7}$ K. F. Li, D. S. Ong, J. P. R. David, G. J. Rees, R. C. Tozer, P. N. Robson, and R. Grey, IEEE Trans. Electron Devices 45, 2102 (1998).

${ }^{8}$ G. M. Dunn, G. J. Rees, J. P. R. David, S. A. Plimmer, and D. C. Herbert, Semicond. Sci. Technol. 12, 111 (1997).

${ }^{9}$ F. Ma, S. Wang, X. Li, K. A. Anselm, X. G. Zheng, A. L. Holmes, and J. C. Campbell, J. Appl. Phys. 92, 4791, (2002).

${ }^{10}$ D. Vasileska and S. M. Goodnick, Mater. Sci. Eng., R. 38, 181 (2002).

${ }^{11}$ B. K. Ridley, Quantum Processes in Semiconductors, 4th ed. (Oxford University Press, Oxford, 1999).

${ }^{12}$ K. Kunihiro, K. Kasahara, Y. Takahashi, and Y. Ohno, IEEE Electron Device Lett. 20, 608 (1999).

${ }^{13}$ D. K. Ferry, Semiconductor Transport (Taylor and Francis, London, 2000).

${ }^{14}$ C. Bulutay, Semicond. Sci. Technol. 17, L59 (2002).

${ }^{15}$ M. A. Osman and D. K. Ferry, Phys. Rev. B 36, 6018 (1987).

${ }^{16}$ C. Bulutay, B. K. Ridley, and N. A. Zakhleniuk, Phys. Rev. B 62, 15754 (2000).

${ }^{17}$ C. Bulutay, B. K. Ridley, and N. A. Zakhleniuk, Physica B 314, 63 (2002).

${ }^{18}$ G. Lehmann and M. Taut, Phys. Status Solidi B 54, 469 (1972).

${ }^{19}$ M. V. Fischetti and J. M. Higman, Monte Carlo Device Simulation: Full Band and Beyond, edited by K. Hess (Kluwer, Dordrecht, 1991).

${ }^{20}$ I. H. Oğuzman, J. Kolnik, and K. F. Brennan, J. Appl. Phys. 80, 8 (1996).

${ }^{21}$ R. W. Hockney and J. W. Eastwood, Computer Simulation Using Particles (Institute of Physics, Bristol, 1988), p. 142.

${ }^{22}$ C. Sevik and C. Bulutay, IEE Proc.-J: Optoelectron. 150, 86 (2003). 